\title{
ㄷำ1
}

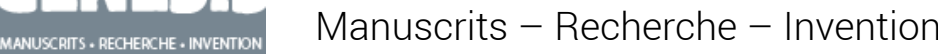

43 | 2016

Bande dessinée

\section{La Bête est morte ! de Calvo}

ou la précision visionnaire des esquisses

Jean-Pierre Mercier

\section{(2) OpenEdition}

Journals

Édition électronique

URL : http://journals.openedition.org/genesis/1712

DOI : 10.4000/genesis. 1712

ISSN : 2268-1590

Éditeur :

Presses universitaires de Paris Sorbonne (PUPS), Société internationale de génétique artistique littéraire et scientifique (SIGALES)

\section{Édition imprimée}

Date de publication : 12 décembre 2016

Pagination : 175-181

ISBN : 9791023105490

ISSN : $1167-5101$

Référence électronique

Jean-Pierre Mercier, "La Bête est morte ! de Calvo », Genesis [En ligne], 43 | 2016, mis en ligne le 04 décembre 2017, consulté le 01 mai 2019. URL : http://journals.openedition.org/genesis/1712 ; DOI : 10.4000/genesis. 1712 


\title{
La Bête est morte! de Calvo ou la précision visionnaire des esquisses
}

\author{
Jean-Pierre Mercier
}

$\mathrm{L}$ a date précise à laquelle Edmond-François Calvo (1892-1957) a commencé à travailler sur La Bête est morte! n'est pas connue, même si certaines sources indiquent 1942. On sait qu'il a élaboré cette œuvre majeure en travaillant à partir des textes fournis par Victor Dancette, son éditeur chez G.P.

À cette époque, Calvo est un auteur relativement nouveau dans le champ de la bande dessinée pour enfants. Il a commencé à fournir des pages aux éditeurs parisiens à partir de 1937 et a rapidement multiplié les collaborations, les éditeurs ayant remarqué et apprécié sa prolixité, et l'engouement qu'il suscitait chez les jeunes lecteurs.

Considéré comme un maître de la bande dessinée animalière, Calvo est un héritier de Samivel (le dessinateur de livres pour enfants comme Brun l'ours plus que le grand illustrateur de la montagne), d'Albert Dubout (pour le goût des compositions grouillantes de détails) et d'illustrateurs du XIXe siècle tels que Grandville, Doré, Sullivant, Kley ou encore le trop méconnu Félix Lorioux. Et il a bien sûr subi l'influence de Walt Disney, qui a génialement redéfini le genre animalier dans ses premiers dessins animés à partir de 1927 et marqué de son empreinte non seulement le dessin animé mais également la bande dessinée du siècle dernier.

Travailleur acharné, que sa fille Anny décrivait dans les années 1990 comme littéralement enchaîné à sa table à dessin, Calvo a dessiné La Bête... clandestinement, en plus des nombreuses pages qu'il fournissait chaque semaine aux éditeurs parisiens. Si l'on ne sait pas quand il a débuté, on sait quand il a terminé, puisque le premier volume de ce récit initialement prévu en deux tomes a paru en 1944 et le second en 1945.

Une péripétie bien connue des amateurs, et qui fait aujourd'hui le bonheur des collectionneurs, permet d'inférer que la réalisation des quarante pages du deuxième tome a dû être faite assez tardivement. En effet, la parution du tome I en 1944 a provoqué une vive réaction des studios Disney de Burbank, qui ont adressé aux éditions G.P. une missive rédigée en anglais et lourde de menaces. Les membres de la maison mère de Mickey et Donald voyaient en effet d'un mauvais œil que le loup censé représenter Hitler dans La Bête... ait, selon eux, un air de ressemblance bien trop marqué avec le loup dont ils avaient fait le héros de leur propre dessin animé de propagande antinazie, Der Fuehrer's Face (1943). Inquiet de cette menace, l'éditeur contraignit Calvo à modifier la physionomie de son loup. Il fendit donc toutes les truffes, non seulement celle du loup représentant Hitler, mais aussi de tous les loups qui grouillent dans les quatre-vingts pages de l'histoire. On voit clairement dans les pages du tome I que la modification a été faite sur des dessins déjà réalisés, alors que les truffes du tome II ont vraisemblablement été directement fendues par Calvo, si l'on peut dire. On peut donc en déduire que les pages du tome II ont été finalisées après la parution du tome I, soit en 1945.

Le succès de La Bête... à sa parution a été massif, même si l'on manque de données chiffrées pour mesurer l'impact d'une œuvre publiée à une époque où la France était en proie à de multiples pénuries, dont une sévère pénurie de papier. Dancette, Calvo et l'éditeur durent bénéficier d'appuis, car les éditions originales sont de très bonne qualité, de papier, comme d'impression et de reliure. On sait cependant, grâce à l'incident des truffes, qu'il y eut au moins deux tirages du tome I, puis un tirage ultérieur d'un recueil rassemblant les deux tomes en un seul volume. On sait également que ce recueil fut également édité dans une version anglaise (The Beast Is Dead!, 1946) et hollandaise (Het Beest ist Dood, 1946). On peut donc en déduire que plusieurs dizaines de milliers d'exemplaires de ces premières éditions se sont écoulées dans les premières années de l'immédiat aprèsguerre, sans pouvoir hélas être plus précis.

Alors que Calvo faisait, comme la plupart des dessinateurs de son époque, peu de cas de ses originaux (beaucoup ont été perdus et ceux qu'il avait conservés, plusieurs 
centaines tout de même, étaient entreposés sans trop de ménagement au bas d'une armoire dans l'habitation qu'il occupait à Paris), il a réservé un traitement particulier aux originaux de La Bête est morte.

Il les a confiés à un relieur qui a fixé ces planches sur d'épaisses feuilles grises, elles-mêmes fixées sur charnières et reliées sous une couverture en cuir, le dos de la reliure étant orné du titre de l'ouvrage, gravé à l'or fin. Le résultat ressemble à un livre géant 1 , qui pèse son poids.

Avec La Bête..., Calvo était conscient d'avoir accompli un chef-d'œuvre et souhaitait manifestement à la fois le préserver et pouvoir en disposer commodément.

Jusqu'à une date récente, on ne disposait d'aucun document permettant d'éclairer les conditions de l'élaboration de cette œuvre majeure. Or, il se trouve que parmi les pages de Calvo que le musée de la Bande dessinée a ces dernières années acquises auprès de la famille de l'artiste, figure la quasi-intégralité des pages d'un magnifique abécédaire (Monsieur Loyal présente, 1946). Une demi-douzaine de ces pages sont en fait des dessins originaux qui ont été découpés et recollés sur des feuilles de dessin, vraisemblablement par Calvo lui-même. Ces pages de support ont elles-mêmes été découpées sans trop d'égards. Au verso de celles-ci se trouvent des crayonnés. Si l'un de ces crayonnés n'est que l'esquisse de cases entièrement vides, toutes les autres comportent des dessins très poussés où se reconnaît sans aucun doute la patte de Calvo.
Mieux, le lecteur aguerri des pages imprimées de La Bête est morte! identifie sans peine telle case extraite de telle page, telle autre case extraite de telle autre page... Ces esquisses miraculeusement rescapées de la destruction permettent de renseigner le processus créatif d'un des grands maîtres de la bande dessinée mondiale.

On constate ainsi qu'au stade préliminaire de la mise en place et des croquis, Calvo «poussait» son travail si loin qu'on identifie immédiatement le dessin final en couleurs dans le dessin préparatoire au crayon de papier. Le trait de crayon pousse loin les postures, les expressions. Il manque la rondeur qu'apportera ensuite le travail au pinceau, dont Calvo maîtrise souverainement les effets de pleins et de déliés. Mais on y gagne la délicatesse d'un trait dont la souple précision enchante. La plupart des dessinateurs reconnaissent que quelque chose se perd dans le passage du crayonné à l'encrage : une spontanéité, une vibration s'évaporent, en quelque sorte. Le trait repassé à l'encre s'épaissit, se raidit, dans bien des cas. Comme on le voit, ce n'est pas vrai pour Calvo qui sait conserver, d'un outil l'autre, l'élan et la finesse de son expression graphique.

1. Les planches originales des Aventures de Rosalie (1947), autre chefd'œuvre de Calvo, ont subi le même traitement et sont rassemblées dans une reliure qui a été acquise par le musée de la Bande dessinée d'Angoulême. 


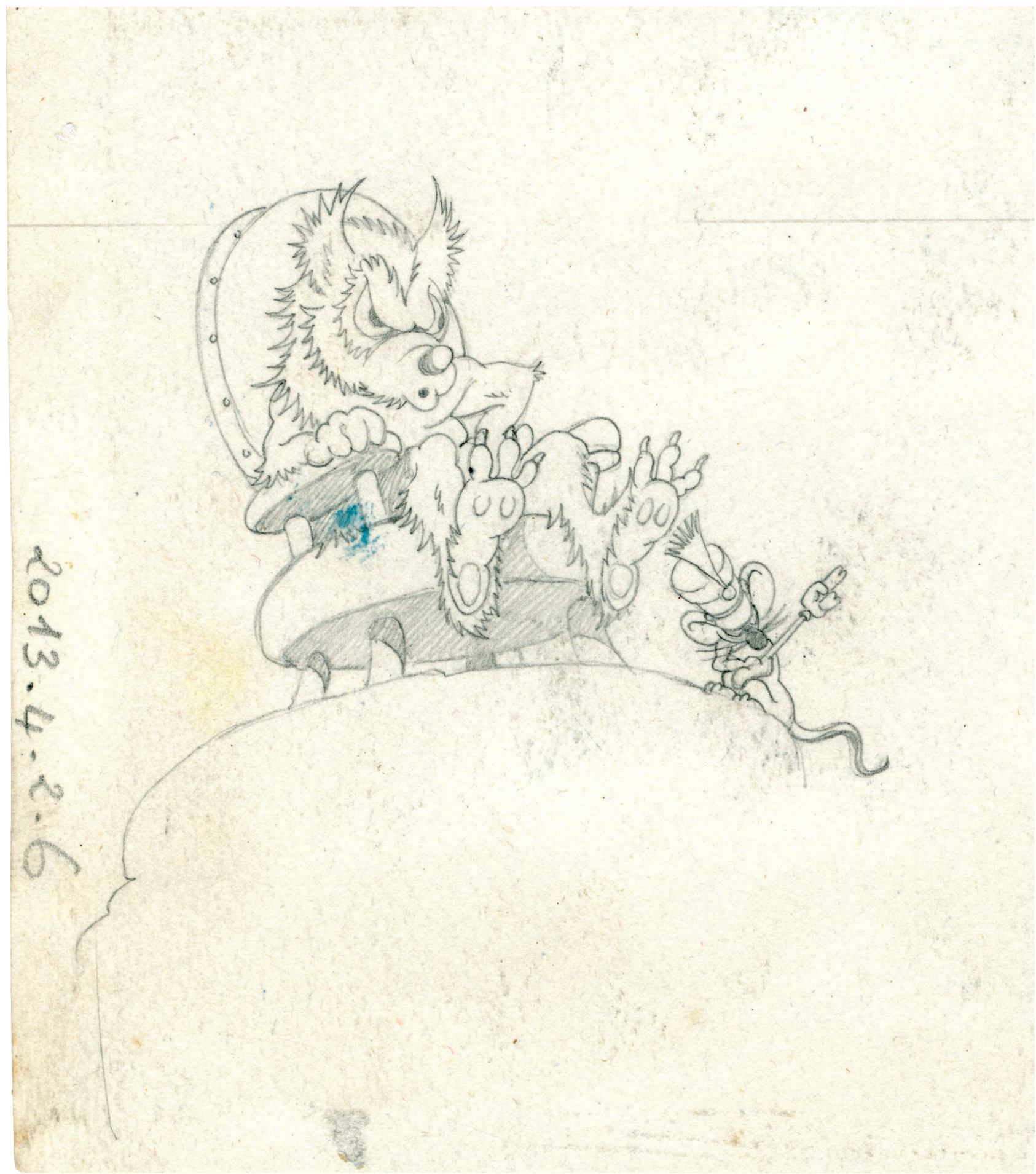

Fig. 1 : Calvo, esquisse au dos des planches de Monsieur Loyal présente, vers 1945 (musée de la Bande dessinée d'Angoulême) 


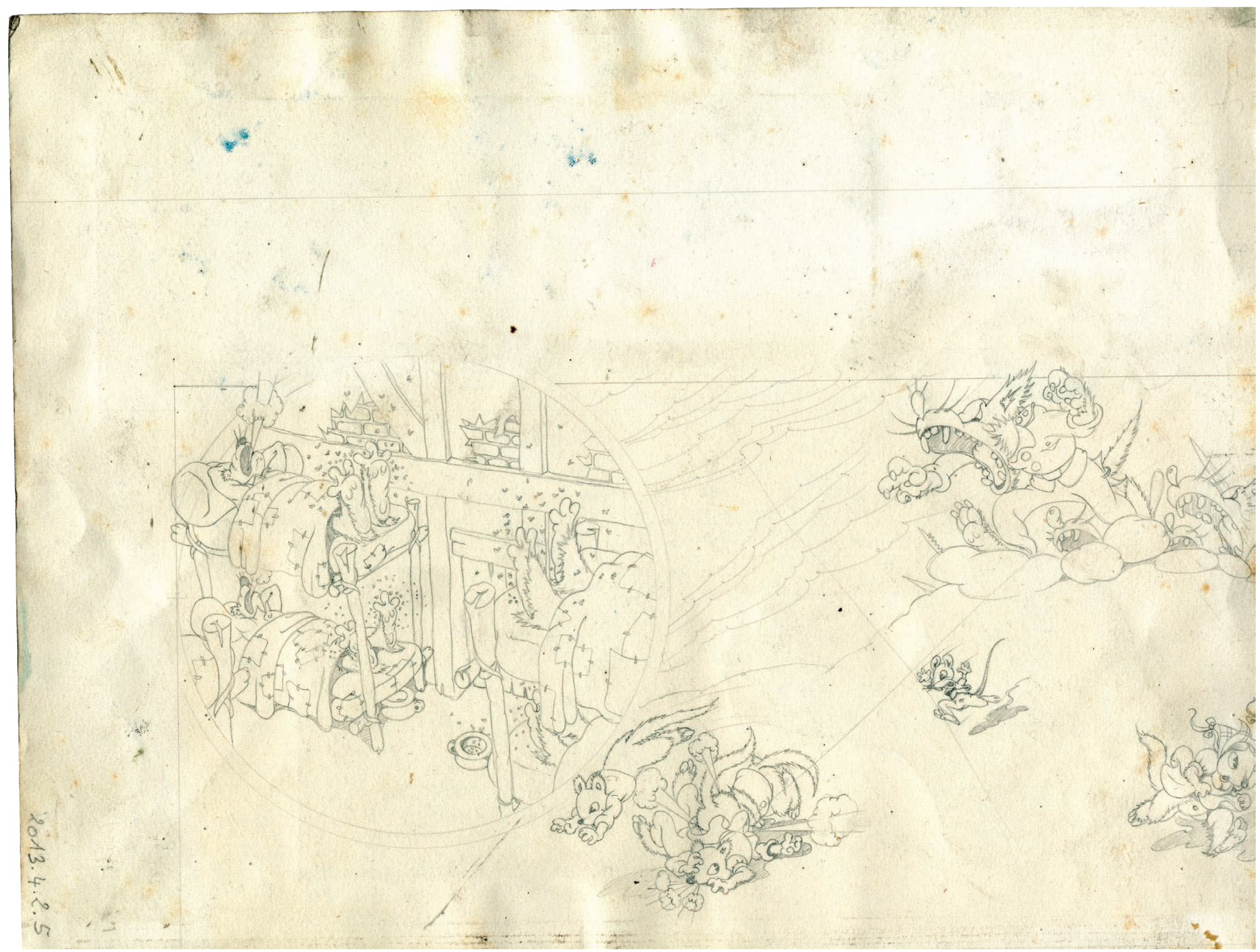

Fig. 2 : Calvo, esquisse au dos des planches de Monsieur Loyal présente, musée de la bande dessinée, vers 1945. À gauche, une case circulaire dont le travail de composition est achevé. Â droite, une intuition graphique très aboutie qui évoluera vers une composition plus complexe (voir ci-dessous).

Fig. 3 (page de droite en haut) : État final de la planche correspondant à la partie gauche du crayonné (fig. 2), La Bête est morte!, 1945, tome I. On notera la conservation de tous les éléments de l'esquisse, déjà très aboutie, la présence dès l'origine de la «case circulaire » insérée dans le flux séquentiel du gaufrier, l'ajout de quelques dizaines de mouches supplémentaires. L'encrage et la mise en couleurs (gouache et aquarelle) créent les reliefs et accentuent la perspective verticale légèrement désaxée (dont on peut suivre les lignes de fuite en longeant les montants des lits) et mettent en contraste la douceur nocturne des couleurs (anticipation des techniques de «nuit américaine» avec les émanations comiques des personnages).

Fig. 4 (page de droite en bas) : État final de la planche dans La Bête est morte!, t. I, 1945, correspondant à la partie droite du crayonné ci-dessus. On notera l'impressionnante conversion du Loup seul et simple en blindé complexe dirigé par le Loup (avec dédoublement), le changement d'expression qui fait évoluer le Loup à la gueule dévorante grande ouverte en Loup ironique et cruel, mais la conservation du principe de composition de la chargemassacre, de la précipitation et de l'écrasement des écureuils par l'effet de pente, dont on peut anticiper mentalement les dégâts. Le travail à peine esquissé des nuages dans le croquis initial, avec un bel effet dynamique qui suit la pente du monticule, se traduit dans la version finale par un ciel repensé à l'horizontale et traité finement à l'aquarelle (dégradés de bleu et de rouge), créant une stabilité tragique qui met en valeur la future et lourde bascule du tank vers l'avant. 

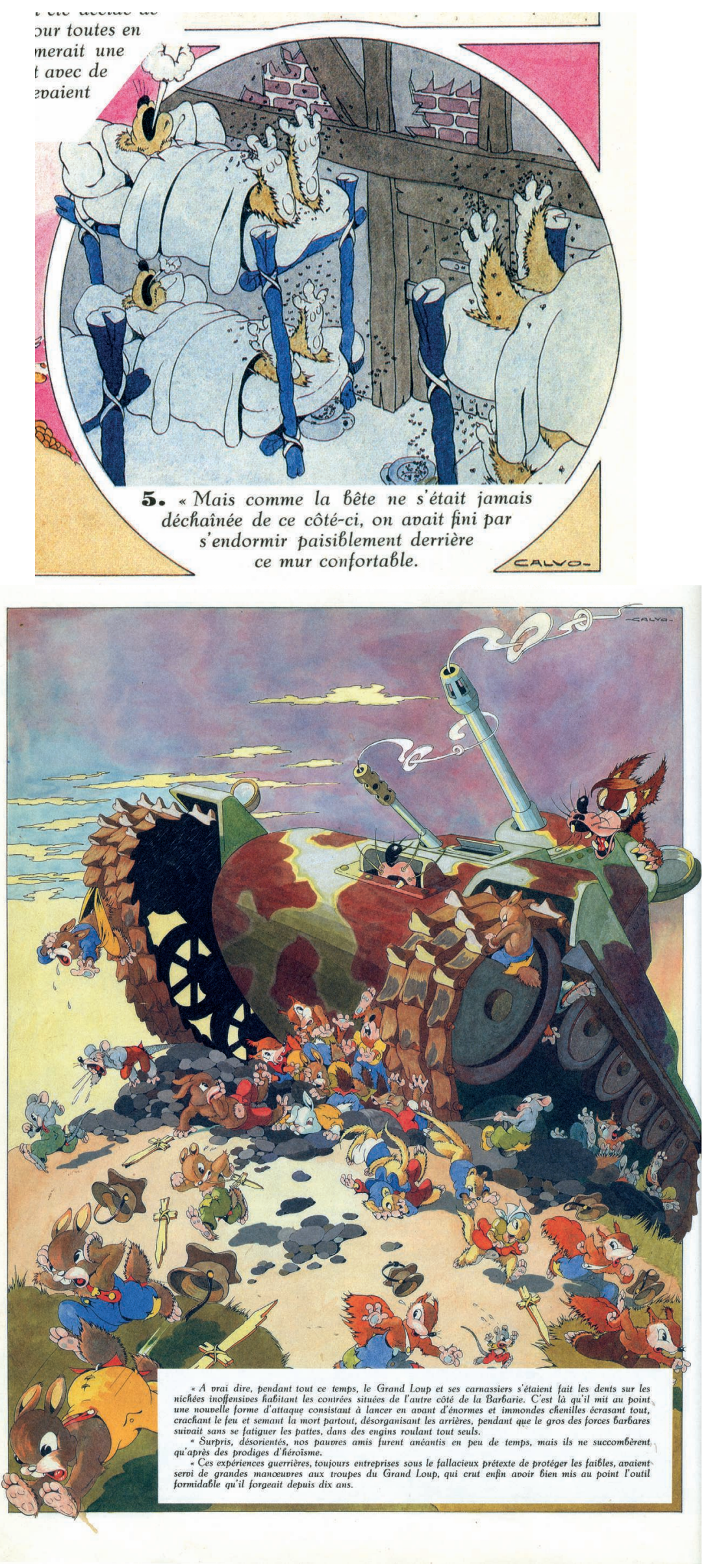


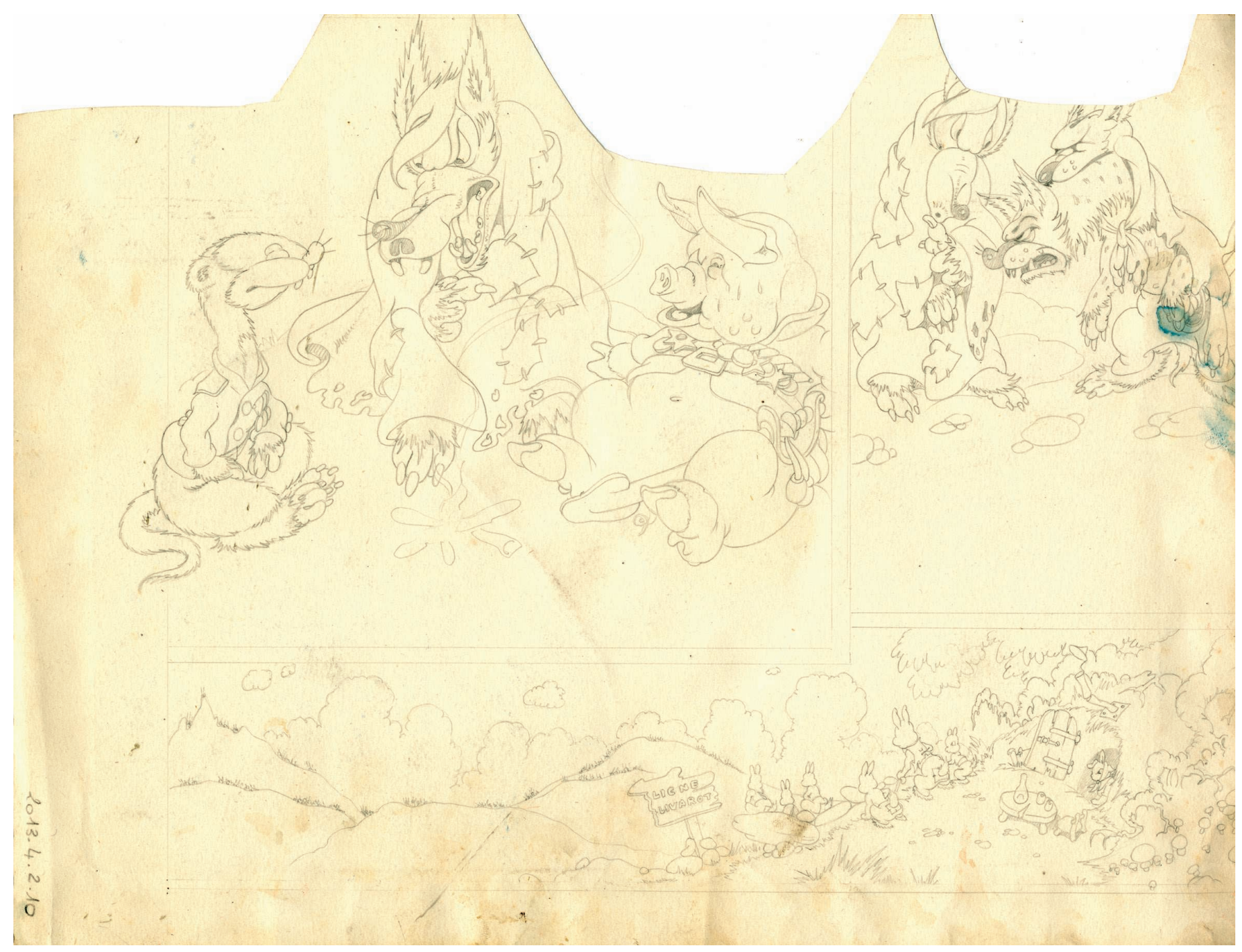

Fig. 5 : Calvo, esquisse au dos des planches de Monsieur Loyal présente, musée de la bande dessinée, vers 1945.

La précision du trait sur les deux esquisses du haut semble interdire l'idée d'un «rough», d'un brouillon.

Il s'agit bien pourtant d'un état préparatoire d'une case où les expressions du loup vont notablement évoluer (voir fig. 6). 


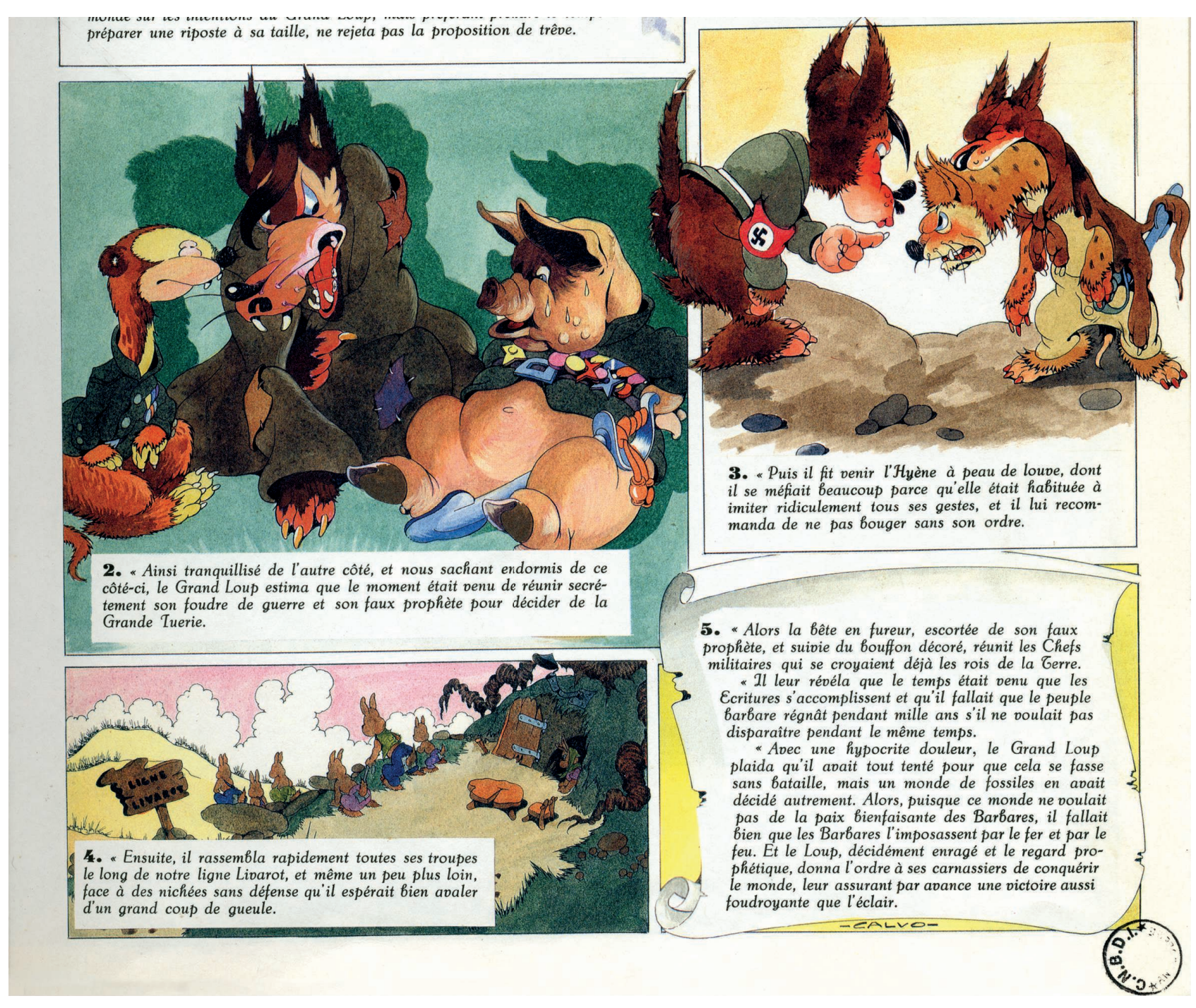

Fig. 6 : État final de la planche. On constate l'introduction d'un soupçon d'angoisse et de folie dans le regard du Loup, plus simplement cantonné dans les esquisses à l'expression de la terreur. C'est une variation notable sur l'ensemble de l'album où les expressions du Loup ont été travaillées par Calvo dans le sens de l'émotionnel, de l'irrationnel et de l'inconscience. 